\title{
Constraining $C P$ violation in neutral meson mixing with theory input
}

\section{Sascha Turczyk*}

Ernest Orlando Lawrence Berkeley National Laboratory, University of California, Berkeley, CA 94720

E-mail: ssturczyk@lbl.gov

\section{Marat Freytsis}

Ernest Orlando Lawrence Berkeley National Laboratory, University of California, Berkeley, CA 94720

Berkeley Center for Theoretical Physics, Department of Physics, University of California, Berkeley, CA 94720 E-mail: freytsisaberkeley • edu

\section{Zoltan Ligeti}

Ernest Orlando Lawrence Berkeley National Laboratory, University of California, Berkeley, CA 94720

E-mail: ligeti@lbl.gov

\begin{abstract}
There has been a lot of recent interest in experimental hints of $C P$ violation in $B_{d, s}^{0}$ mixing. The $\mathrm{D} \varnothing$ measurement of the semileptonic $C P$ asymmetry would - with higher significance - be a clear signal of beyond the standard model physics. In this talk I present a relation [1] for the mixing parameters, which allows clearer interpretation of the data in models in which new physics enters in $M_{12}$ and/or $\Gamma_{12}$. This result implies that the central value of the $\mathrm{D} \varnothing$ measurement in $B_{d, s}^{0}$ decay is not only in conflict with the standard model, but in a stronger tension with data on $\Delta \Gamma_{s}$ than previously appreciated. After I derive the relation between the theoretical prediction of $\left|\Gamma_{12}\right|$ and the measurements of $\Delta M, \Delta \Gamma$ and $A_{\mathrm{SL}}$, I will explain how this result can help to better constrain $\Delta \Gamma$ or $A_{\mathrm{SL}}$, whichever is less precisely measured.
\end{abstract}

36th International Conference on High Energy Physics,

July 4-11, 2012

Melbourne, Australia

${ }^{*}$ Speaker. 


\section{Introduction}

The $\mathrm{D} \emptyset$ measurement of the $C P$ asymmetry in decays of a $b \bar{b}$ pair to two same-sign muons [2] hinted towards $C P$ violation in $B-\bar{B}$ mixing, which would be a clear sign of new physics (NP) $[3,4]$

$$
A_{\mathrm{SL}}^{b}=-[7.87 \pm 1.72 \text { (stat) } \pm 0.93 \text { (syst) }] \times 10^{-3} .
$$

This is a linear combination of the two individual asymmetries, as both $B_{d}^{0}$ and $B_{s}^{0}$ are produced

$$
A_{\mathrm{SL}}^{b}=(0.594 \pm 0.022) A_{\mathrm{SL}}^{d}+(0.406 \pm 0.022) A_{\mathrm{SL}}^{s} \text {. }
$$

The time evolution of the flavor eigenstates is determined by

$$
i \frac{\mathrm{d}}{\mathrm{d} t}\left(\begin{array}{l}
\left|B^{0}(t)\right\rangle \\
\left|\bar{B}^{0}(t)\right\rangle
\end{array}\right)=\left(M-\frac{i}{2} \Gamma\right)\left(\begin{array}{l}
\left|B^{0}(t)\right\rangle \\
\left|\bar{B}^{0}(t)\right\rangle
\end{array}\right),
$$

$M$ and $\Gamma$ are $2 \times 2$ Hermitian matrices, $C P T$ invariance implies $M_{11}=M_{22}$ as well as $\Gamma_{11}=\Gamma_{22}$. The physical eigenstates are given by

$$
\left|B_{H, L}\right\rangle=p\left|B^{0}\right\rangle \mp q\left|\bar{B}^{0}\right\rangle
$$

where we chose $|p|^{2}+|q|^{2}=1$. $C P$ violation in mixing occurs if the mass and $C P$ eigenstates do not coincide, $\delta \equiv\left\langle B_{H} \mid B_{L}\right\rangle=\left(|p|^{2}-|q|^{2}\right) /\left(|p|^{2}+|q|^{2}\right) \neq 0$. The solution for the mixing parameters satisfies the relation $q^{2} / p^{2}=\left(2 M_{12}^{*}-i \Gamma_{12}^{*}\right) /\left(2 M_{12}-i \Gamma_{12}\right)$. In the small $\delta$ limit, $A_{\mathrm{SL}} \approx 2 \delta$ is a very good approximation in the $B_{d / s}$-systems. In the $\left|\Gamma_{12} / M_{12}\right| \ll 1$ limit we have for the mixing parameters

$$
\Delta m \approx 2\left|M_{12}\right|, \quad \Delta \Gamma \approx 2\left|\Gamma_{12}\right| \cos \left[\arg \left(-\Gamma_{12} / M_{12}\right)\right], \quad A_{\mathrm{SL}} \approx \operatorname{Im}\left(\Gamma_{12} / M_{12}\right) .
$$

In this limit, which applies model independently for $B_{d, s}^{0}$ systems, $q / p$ is a pure phase to a good approximation, determined by $M_{12}$, which has good a sensitivity to NP. The width difference $\Delta \Gamma_{s} \equiv$ $\Gamma_{L}-\Gamma_{H}$ has been measured experimentally in the $B_{s}$ system, and has not been observed for the $B_{d}$ meson. We use the most precise single measurement from LHCb [5] in the lack of a world average

$$
\Delta \Gamma_{s}=(0.116 \pm 0.019) \mathrm{ps}^{-1} .
$$

For $\Delta m_{s}$ we take the average of the CDF [6] and LHCb [7, 8] measurements, $\Delta m_{d}$ from HFAG [9]

$$
\Delta m_{s} \equiv m_{H}-m_{L}=(17.719 \pm 0.043) \mathrm{ps}^{-1}, \quad \Delta m_{d}=(0.507 \pm 0.004) \mathrm{ps}^{-1} .
$$

The individual asymmetries measurements at the $B$ factories [9] and at $\mathrm{D} \varnothing[10]$ are compatible with the Standard Model $(\mathrm{SM})$ prediction[11] $A_{\mathrm{SL}}^{d}=-(0.5 \pm 5.6) \times 10^{-3}, A_{\mathrm{SL}}^{s}=-(1.7 \pm 9.2) \times 10^{-3}$. In the following we clarify, if there are any non-trivial constraints on the mixing parameters, besides the requirement of having positive mass and width eigenvalues for the physical states.

\section{Theoretical Constraints on the Mixing Parameters}

The unitarity bound $[12,13]$ is a requirement on the mixing parameters, which constrains the eigenvalues of $\Gamma$ to be positive independent of the physical eigenvalues, or equivalently

$$
\delta^{2}<\frac{\Gamma_{H} \Gamma_{L}}{\left(m_{H}-m_{L}\right)^{2}+\left(\Gamma_{H}+\Gamma_{L}\right)^{2} / 4}=\frac{1-y^{2}}{1+x^{2}} .
$$

Here we define, using $\Gamma=\left(\Gamma_{H}+\Gamma_{L}\right) / 2$, the quantities $x=\left(m_{H}-m_{L}\right) / \Gamma$ and $y=\left(\Gamma_{L}-\Gamma_{H}\right) /(2 \Gamma) . x$ is positive by definition, while $y \in(-1,+1)$. For the derivation we define the complex quantities

$$
a_{i}=\sqrt{2 \pi \rho_{i}}\left\langle f_{i}|\mathscr{H}| B\right\rangle, \quad \bar{a}_{i}=\sqrt{2 \pi \rho_{i}}\left\langle f_{i}|\mathscr{H}| \bar{B}\right\rangle,
$$


with $\rho_{i}$ denoting the phase space density for the final state $f_{i}$. If we treat $a_{i}$ and $\bar{a}_{i}$ as vectors in a complex $N$-dimensional vector space, then taking the standard inner product on complex vector spaces, and using the optical theorem [12], amounts to the relations

$$
a_{i}^{*} a_{i}=\Gamma_{11}, \quad \bar{a}_{i}^{*} \bar{a}_{i}=\Gamma_{22}, \quad \bar{a}_{i}^{*} a_{i}=\Gamma_{12},
$$

where $C P T$ fixes $\Gamma_{11}=\Gamma_{22}=\Gamma$. Applying the Cauchy-Schwarz inequality to $\left|\left(\bar{a}_{i}\right)\right|$ in (2.3) implies [12]

$$
\left|\Gamma_{12}\right| \leq \Gamma_{11} .
$$

Thus the eigenvalues of the $\Gamma$ matrix must be positive in addition to the physical width $\Gamma_{H, L}>0$. To see that this is also equivalent to the unitarity bound of Eq. (2.1), we use Eq. (1.4) to define $a_{H, L}$ analogously to the physical states, and proceed with similar steps as above. The unitarity bound in Eq. (2.1) then arises from using the resulting expressions with the relations (2.3) in Eq. (2.4).

\subsection{Deriving a Relation using Theoretical Input}

In the kaon system, for which this bound was originally derived, using the inequality in Eq. (2.4) was a necessity due to the dominance of long-distance physics in the result. For $B_{d, s}$ mesons, the large mass scale $m_{b} \gg \Lambda_{\mathrm{QCD}}$ allows $\Gamma_{11}$ and $\Gamma_{12}$ to be calculated in an operator product expansion, and at leading order $\left|\Gamma_{12} / \Gamma_{11}\right|=\mathscr{O}\left[\left(\Lambda_{\mathrm{QCD}} / m_{b}\right)^{3}\left(16 \pi^{2}\right)\right]$. We extend the preceding derivation with assuming additional theoretical knowledge in Eq. (2.4), and define

$$
y_{12}=\left|\Gamma_{12}\right| / \Gamma .
$$

Thus we obtain as the solution an exact relation instead of the inequality

$$
\delta^{2}=\frac{y_{12}^{2}-y^{2}}{y_{12}^{2}+x^{2}}=\frac{\left|\Gamma_{12}\right|^{2}-(\Delta \Gamma)^{2} / 4}{\left|\Gamma_{12}\right|^{2}+(\Delta m)^{2}} .
$$

This equation also follows from the solution of the eigenvalue problem, previously derived in Ref. [14] with the resulting bound on $\delta$ noted. It also appears in related forms in Refs. [15, 16] and follows from Eqs. (9) and (12) in [17]. For fixed $x$ and $y, \delta^{2}$ is monotonic in $y_{12}$ : an upper bound on $y_{12}$ gives an upper bound on $|\delta|$. With the requirement $y_{12} \leq 1$ the usual unitarity bound in Eq. (2.1) is recovered. A better understanding of the physical situation can be gained, by obtaining Eq. (2.6) from a scaling argument: $\delta$ only depends on mixing parameters, independent of $\Gamma$. One can then scale $\Gamma$ by $y_{12}$, which cannot affect $\delta$ but changes $x \rightarrow x / y_{12}$ and $y \rightarrow y / y_{12}$. The exact relation Eq. (2.6) follows then from this argument and Eq. (2.1). The derivation above makes the physical origin of this relation clear and also holds in the $C P T$ violating case for $|\delta|^{2}$, as $\delta$ can become complex in this case. Even if a precise calculation of $\Gamma_{12}$ is not possible or one assigns a very conservative uncertainty to it, an upper bound on $y_{12}$ implies an upper bound on $|\delta|$, stronger than Eq. (2.1). For small values of $y_{12}$, as in the $B_{d}$ system, this bound can be much stronger.

\subsection{Application to Recent Data}

First we will compare the relation using the measured mass difference and theory prediction of $\left|\Gamma_{12}\right|$ to the result in Eq. (1.1) from D $\varnothing$ and then to the individual asymmetries. We can only compare the absolute value of $A_{\mathrm{SL}}^{b}$ measured by $\mathrm{D} \emptyset$ with the result implied by the relation above, since Eq. (2.6) only bounds $|\delta|$. Thus the bound on $A_{\mathrm{SL}}^{b}$ is not sensitive to possible cancellations between $A_{\mathrm{SL}}^{d}$ and $A_{\mathrm{SL}}^{s}$. Denoting this upper bound by $\delta_{\max }^{d, s}$ and using the weight factors from Eq. (1.2), 


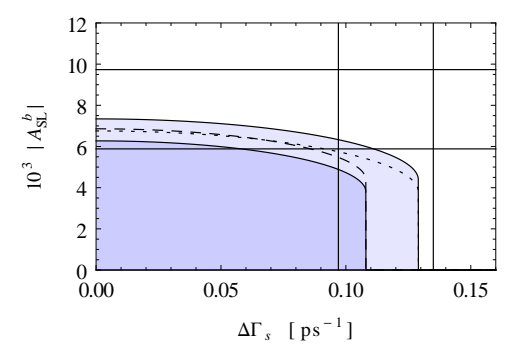

Figure 1: Upper bounds on $A_{\mathrm{SL}}^{b}$ as a function of $\Delta \Gamma_{s}$, setting $\Delta \Gamma_{d}=0$, description is in text.

$\left|A_{\mathrm{SL}}^{b}\right| \leq(1.188 \pm 0.044) \delta_{\max }^{d}+(0.812 \pm 0.044) \delta_{\max }^{s}$. As $\Delta m_{d, s}$ are precisely known, we plot the bound as a function of the width differences $\Delta \Gamma_{d, s}$. Because $\Delta \Gamma_{d}$ has not been measured yet, we set this to zero as the most conservative choice. If LHCb measures the difference $A_{\mathrm{SL}}^{s}-A_{\mathrm{SL}}^{d}$ [18], then the above bound with modified coefficients apply for that measurement as well. In Fig. 1, the darker shaded region shows the upper bound on $\left|A_{\mathrm{SL}}^{b}\right|$ using the $1 \sigma$ ranges for $\left|\Gamma_{12}^{d, s}\right|$ in the SM [11], and the lighter shaded region includes both $2 \sigma$ regions, with

$$
2\left|\Gamma_{12}^{s}\right|=(0.087 \pm 0.021) \mathrm{ps}^{-1} \quad \text { and } \quad 2\left|\Gamma_{12}^{d}\right|=(2.74 \pm 0.51) \times 10^{-3} \mathrm{ps}^{-1} .
$$

The dashed [dotted] curve shows the impact of using the $2 \sigma$ region for $\Gamma_{12}^{d}\left[\Gamma_{12}^{s}\right]$ The vertical boundaries of the shaded regions arise because $\left|\Delta \Gamma_{s}\right|>2\left|\Gamma_{12}^{s}\right|$ is unphysical. A tension between the $A_{\mathrm{SL}}^{b}$ measurement and the bound is visible, independent of the discrepancy between the $\mathrm{D} \emptyset$ result and the global fit to the latest available experimental data [19]. Of course, independent of the D $\varnothing$
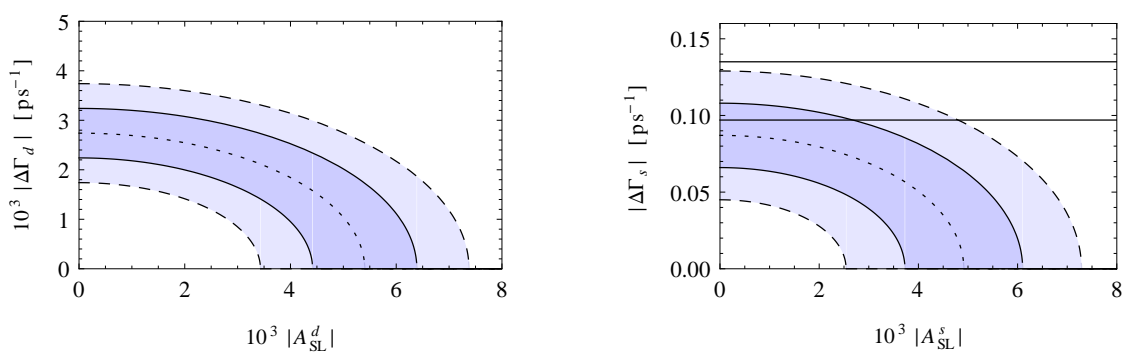

Figure 2: Left plot: the region allowed by Eq. (2.6) in the $A_{\mathrm{SL}}^{d}-\Delta \Gamma_{d}$ plane. The SM calculation of $\left|\Gamma_{12}^{d, s}\right|$ at $1 \sigma[2 \sigma]$ gives the darker [lighter] shaded region. Right plot: same for $A_{\mathrm{SL}}^{s}-\Delta \Gamma_{s}$; the straight lines show the $1 \sigma$ range of the $\mathrm{LHCb}$ result for $\Delta \Gamma_{s}$.

measurement of $A_{\mathrm{SL}}^{b}$, we can also compare the bound implied by our relation to the individual best bounds on the semi-leptonic asymmetries. To this end, in Fig. 2 we plot $A_{\mathrm{SL}}^{d}$ vs. $\Delta \Gamma_{d}$ (and similarly for $B_{s}$ ) allowed by Eq. (2.6) and the $1 \sigma$ and $2 \sigma$ ranges of the SM calculation of $\left|\Gamma_{12}\right|$ [11]. Here, there have been no discrepancies claimed between the theory predictions and measurements, but our relation allows us to place a bound tighter than the current experimental constraints which is more robust than the purely theoretical SM calculation as outlined above. A non-zero observation of $\Delta \Gamma_{d}$ will strengthen the upper bound on $A_{\mathrm{SL}}^{d}$ obtained with the relation (2.6).

\subsection{No-Go theorem}

We will show, that besides the positivity conditions on the physical mixing parameters $M_{H, L}$, 
$\Gamma_{H, L}$, algebraical relations and the unitarity bound no other consistency relations from physical considerations can appear. The no-go theorem does not apply for the relation presented in this talk, because we additionally assume knowledge about a parameter of the underlying Hamiltonian. We now sketch a physical understanding for the proof of this theorem. From Eqs. (2.3) and (2.4) it is obvious that the unitarity bound is exactly saturated, if the two vectors of $B_{0}$ and $\bar{B}_{0}$, equivalently $B_{L}$ and $B_{H}$, are aligned: $\left\langle f|\mathrm{~T}| B_{H}\right\rangle \propto\left\langle f|\mathrm{~T}| B_{L}\right\rangle$. Without loss of generality we can start with an arbitrary, generic decaying two-state system in the Wigner-Weisskopf approximation, i.e. no strong interactions obscure the situation. We therefore choose an orthogonal, non $C P$ violating system, which obeys the above required alignment of states as a starting point, which has $\delta \equiv 0$. By adding arbitrary new ultraviolet (UV) physics, which does not necessarily need to be compatible with data, we can change $M_{12}$ independently of $\Gamma_{12}$, introducing a non-vanishing $\delta$. We can then vary $M_{12}$, however have to keep the mass and width of the physical states positive. We can always saturate the unitarity bound, leaving no room for stronger constraints than the unitarity bound in any parameter space. In other words by relaxing the constraint of having no $C P$ violation $\operatorname{Arg} M_{12}=\operatorname{Arg} \Gamma_{12}$, this relation gets replaced by a new constraint, the unitarity bound. Thus the total number of relations is conserved and without assuming knowledge no further bound or relation can be obtained.

\section{Discussion}

We derived not an absolute bound in the fashion of the unitarity bound but a relation between calculable and measured quantities. It is thus worth clarifying the relationship of our result to the stated 3.9 $\sigma$ disagreement of $A_{\mathrm{SL}}^{b}$ with the SM reported in [2]. The SM prediction of $A_{\mathrm{SL}}$ uses the calculation of $\Gamma_{12}$, and $\left|\Gamma_{12}\right|$ also enters our bound; the discrepancies are thus correlated. Although the calculation of $\left|\Gamma_{12}\right|$ and $\operatorname{Im}\left(\Gamma_{12}\right)$ both rely on the same operator product expansion and perturbation theory, the existence of large cancellations in $\operatorname{Im}\left(\Gamma_{12}\right)$ may lead one to think that the uncertainties could be larger in its SM calculation than what is tractable in the behavior of its next-to-leading order calculation $[20,21]$. The sensitivity of $\Gamma_{12}$ to new physics is generally weaker than that of $M_{12}$ (see [22, 23] for other options). Thus, it is interesting to determine $\delta$ from Eq. (2.6), besides its direct calculation. The relation (2.6) is a monotonic function in $y_{12}$ and thus an upper bound on this theory prediction implies an upper bound on $|\delta|$. Therefore this relation is much stronger for small values of $y_{12}$, as is e.g. present in the $B_{d}$ system. A non-zero observation of the width difference does improve the upper bound as well. Now we are in the position to present numerical upper bounds for the individual asymmetries. We use $\Delta \Gamma_{s}$ from LHCb in Eq. (1.6) and neglect $\Delta \Gamma_{d}$ and find the $2 \sigma$ bounds

$$
\left|A_{\mathrm{SL}}^{d}\right|<7.4 \times 10^{-3}, \quad\left|A_{\mathrm{SL}}^{s}\right|<4.2 \times 10^{-3} .
$$

While this bound on $A_{\mathrm{SL}}^{s}$ may seem to disagree with Fig. 2, note that in the plot the uncertainties of $\Gamma_{12}^{s}$ and $\Delta \Gamma_{s}$ are not combined. Propagating the uncertainties, $\left|\Gamma_{12}^{s}\right|^{2}-\left(\Delta \Gamma_{s}\right)^{2} / 4<0$ and thus $\delta^{2}$ is negative at the $1 \sigma$ level, which is an unphysical result. Hence we compute the $2 \sigma$ bounds in Eq. (3.1). The bound on $A_{\mathrm{SL}}^{s}$ is better than the bounds of the measurements in section 1 by more than a factor of 3 , while that for $A_{\mathrm{SL}}^{d}$ is comparable. However, in the case of $B_{d}$ this is driven primarily by the uncertainty in the lifetime difference. If a non-zero value of $\Delta \Gamma_{d}$ were observed, a better bound could be derived. It is worth emphasizing that this implication goes in both directions, 
given that an observation of $A_{\mathrm{SL}}^{d} \neq 0$ may happen before that of $\Delta \Gamma_{d} \neq 0$. Due to Eq. (2.6), as soon as one of the two is measured to be nonzero, the other is constrained to be significantly smaller at worst and given a definite prediction at best.

\section{Acknowledgments}

S.T. thanks the organizers for their effort to host this conference and for providing financial support. ST is supported by a DFG Forschungsstipendium under contract no. TU350/1-1.

\section{References}

[1] M. Freytsis, Z. Ligeti and S. Turczyk, arXiv:1203.3545 [hep-ph].

[2] V. M. Abazov et al. [D0 Collaboration], Phys. Rev. D 84 (2011) 052007 [arXiv:1106.6308 [hep-ex]].

[3] S. Laplace, Z. Ligeti, Y. Nir and G. Perez, Phys. Rev. D 65 (2002) 094040 [hep-ph/0202010].

[4] Z. Ligeti, M. Papucci, G. Perez and J. Zupan, Phys. Rev. Lett. 105 (2010) 131601 [arXiv:1006.0432 [hep-ph]].

[5] LHCb Collaboration, CERN-LHCb-CONF-2012-002. Linked to LHCb-ANA-2012-004.

[6] A. Abulencia et al. [CDF Collaboration], Phys. Rev. Lett. 97 (2006) 242003 [hep-ex/0609040].

[7] LHCb Collaboration, LHCb-CONF-2011-005. Linked to LHCb-ANA-2011-005.

[8] LHCb Collaboration, LHCb-CONF-2011-050. Linked to LHCb-ANA-2011-005.

[9] D. Asner et al. [Heavy Flavor Averaging Group Collaboration], arXiv:1010.1589 [hep-ex].

[10] V. M. Abazov et al. [D0 Collaboration], Phys. Rev. D 82 (2010) 012003 [Erratum-ibid. D 83 (2011) 119901] [arXiv:0904.3907 [hep-ex]].

[11] A. Lenz and U. Nierste, arXiv:1102.4274 [hep-ph].

[12] J.S. Bell, J. Steinberger, Proceedings of the Oxford Int. Conf. on Elementary Particles, Rutherford Laboratory, Chilton, England, 1965, p. 195.

[13] T. D. Lee and L. Wolfenstein, Phys. Rev. 138 (1965) B1490.

[14] G. C. Branco, L. Lavoura, J. P. Silva, Int. Ser. Monogr. Phys. 103, 1-536 (1999).

[15] M. Ciuchini, E. Franco, D. Guadagnoli, V. Lubicz, M. Pierini, V. Porretti and L. Silvestrini, Phys. Lett. B 655 (2007) 162 [hep-ph/0703204].

[16] A. L. Kagan and M. D. Sokoloff, Phys. Rev. D 80 (2009) 076008 [arXiv:0907.3917 [hep-ph]].

[17] Y. Grossman, Y. Nir and G. Perez, Phys. Rev. Lett. 103 (2009) 071602 [arXiv:0904.0305 [hep-ph]].

[18] M. Calvi [LHCb Collaboration], arXiv:1109.0464 [hep-ex].

[19] A. Lenz, U. Nierste, J. Charles, S. Descotes-Genon, H. Lacker, S. Monteil, V. Niess and S. T'Jampens, Phys. Rev. D 86, 033008 (2012) [arXiv:1203.0238 [hep-ph]].

[20] M. Ciuchini, E. Franco, V. Lubicz, F. Mescia and C. Tarantino, JHEP 0308 (2003) 031 [hep-ph/0308029].

[21] M. Beneke, G. Buchalla, A. Lenz and U. Nierste, Phys. Lett. B 576 (2003) 173 [hep-ph/0307344].

[22] Y. Bai and A. E. Nelson, Phys. Rev. D 82 (2010) 114027 [arXiv:1007.0596 [hep-ph]].

[23] C. Bobeth and U. Haisch, arXiv:1109.1826 [hep-ph]. 